\title{
Novel Stem Cells and Nucleic Acid-Based Vaccine Trials Against Viral Outbreak: A Systematic Evaluation During COVID-2019 Pandemic
}

\author{
Chandan Kumar Maurya ${ }^{1} \cdot$ Radhieka Misra $^{2} \cdot$ Praveen Sharma $^{3}$. \\ Neha Singh $^{4} \cdot$ Harshita Awasthi $^{5} \cdot$ Ruchika Agrawal $^{6}$ - Sanjeev Misra ${ }^{7}$. \\ Shailendra Dwivedi ${ }^{1}$ (I)
}

Received: 27 May 2020/Accepted: 17 June 2020/Published online: 10 July 2020

(C) Association of Clinical Biochemists of India 2020

\begin{abstract}
The current Severe Acute Respiratory Syndrome Coronavirus 2 (SARS-CoV-2) outbreak, the cause of coronavirus disease (COVID-19), has influenced health globally. So far, there are no established management options and prophylaxis for those who have been exposed to SARS-CoV-2, and those who develop COVID-19. Documented scientific evidences in similar viral outbreaks in past suggested few therapy regimens. These rather have not shown promising results in management of current pandemic. So, in the current review, we are exploring novel treatment strategies and therapies that are being explored and are in clinical and preclinical stages of research. To explore more about the same, we directed our search towards stem cell based, DNA based, or RNA based vaccines against COVID-19 under development by various universities, institutes or pharmaceutical companies. The
\end{abstract}

Shailendra Dwivedi

tarang2016@gmail.com

1 Department of Biochemistry, All India Institute of Medical Sciences, Gorakhpur 273008, India

2 Era's Lucknow Medical College, and Hospital, Lucknow 226003, India

3 Department of Biochemistry, All India Institute of Medical Sciences, Jodhpur 342005, India

4 Department of Biotechnology, Sam Higginbottom University of Agriculture, Technology and Sciences, Prayagraj 211007, India

5 Department of Biochemistry, Lucknow University Lucknow, Lucknow 226007, India

6 Department of Ear Nose and Throat, All India Institute of Medical Sciences, Gorakhpur 273008, India

7 Department of Surgical Oncology, All India Institute of Medical Sciences, Jodhpur 342005, India current scientific literature and database search were performed by exploring various Trials registry (NIH: https:// clinicaltrials.gov/ and https://www.coronavirus.gov) and Chinese clinical trial registry http://www.chictr.org.cn/) and for preclinical trials various University, Institutions, Pharmaceutical companies websites and news bulletins along with google search were checked routinely from 3rd March 2020 to 16 May 2020. The term "Stem Cell therapy and COVID-19", "Mesenchymal stem cell and corona 2019 virus", "DNA Vaccines and COVID-19, RNA Vaccines and COVID-19" and "Cell-based therapy with SARS-CoV-2, University/Institutions and COVID-19 research" were used. The vaccine trials (Stem Cells/DNA/ RNA) which were cancelled were not included in this review. Similarly, few others like repurposing of drugs, Nano Vaccines, other miscellaneous trials of Herbs, Music therapy etc., were also excluded. In the present review, we have included the various novel therapies like stem cell therapy, DNA or RNA vaccines which are under development and if proven successful may have a lasting impact on the health industry.

Keywords Stem cell based vaccine - DNA vaccine $\cdot$ RNA vaccine $\cdot$ Nucleic acid based vaccines . Viral disease . COVID-19

\section{Introduction}

Current, Severe Acute Respiratory Syndrome Coronavirus 2 (SARS-CoV-2) pandemic, caused by the novel Coronavirus is one of the deadliest virus outbursts of a century. COVID-19 is clinically characterized by difficulty in breathing, fever, and low blood oxygen level. 
The chronology of COVID-19 infection starts when the initial case series was reported in December 2019 [1]. From 18 December 2019, through December 29, 2019, five patients were hospitalized in hospitals of Hubei Province, China with symptoms of atypical pneumonia and acute respiratory distress syndrome caused by unidentified infectious agent. One of these patients succumbed to the disease [2]. Given the rapidity of its spread and atypical symptoms, an outbreak was suspected and contact tracing was done which led the epidemiologists to a wholesale market of seafood and wild animals in Wuhan District of the province. At that point of time it was assumed to be due to any infective agents (virus) which was later identified as a new strain of coronavirus. This new strain was named as 2019-novel coronavirus (2019-nCoV) on 12 January 2020 by the World Health Organization (WHO). WHO officially named the disease caused by this novel virus as Coronavirus Disease 2019 (COVID-19).

The current outbreak of the COVID-19 virus has caused more than 5598 deaths in India (MoHFW, India) and more than 1,931,076 and 6,057,853 peoples have suffered from Severe Acute Respiratory Syndrome Coronavirus 2 (SARS$\mathrm{CoV}-2)$ in India and worldwide respectively till 2nd June $2020[3,4]$. So far, there is no established treatment option or prophylaxis for those who have been exposed to SARS-CoV2 , and those who develop COVID-19. Reviewing the documented scientific evidence of past similar viral outbreaks in various parts of the world helped in trying few therapy regimens initially but were not found effective in managing the present pandemic perfectly given that the present virus seems diverse in structure and characteristics [5].

Clinical presentation of the persons infected with novel coronavirus ranges from asymptomatic subclinical infection to mild pharyngitis to severe lower respiratory tract illness. Fever, cough, shortness of breath or difficulty breathing, chills, repeated shivering with chills, muscle pain, headache, and sore throat, loss of taste or smell are the few known symptoms [4]. These symptoms may appear 2-14 days after exposure to the virus. Despite advancements in science, technology and relentless research by scientific communities all over the world, a comprehensive and compelling management of COVID-19 is still lacking.

As the uncertainties in management of COVID-19 are growing we started our search on what are novel strategies being explored in management of the COVID-19 outbreak? Thus, we have noticed a plethora of case series, clinical trials, preclinical trials and lab researches. Aiming to know the new modes of treatment we restricted our search only to studies on Stem Cell Biology based and Nucleic Acid based vaccines. Thus we explored various Trials registry (NIH: https://clinicaltrials.gov/ and https://www.cor onavirus.gov) and Chinese clinical trial registry http:// www.chictr.org.cn/) and for preclinical trials various
University, Institutions, Pharmaceutical company's websites and news bulletins portals were checked routinely from 3rd March 2020 to 21st May 2020. The proper phrase with Boolean operator "and" were used for searching in scientific database, like "Stem Cell therapy and COVID19", "Mesenchymal stem cell and corona 2019 virus", "DNA Vaccines and COVID-19", "RNA Vaccines and COVID-19" cell-based therapy with SARS-CoV-2, and University/Institutions were used. The trials on above Stem Cells and DNA/RNA vaccines which were cancelled (ChiCTR2000030329, ChiCTR2000030300, ChiCTR2000030224, ChiCTR2000029816, and ChiCTR2000029812), and others like repurposing of drugs, Nano Vaccines, Viral vectors based vaccines, other miscellaneous trials of Herbs, Music therapy etc., were excluded from the review list. The limitations are not so significant as few registered clinical trials have not mentioned their stage of clinical trials as I, II, and III, and all trials have variable no. of subjects. In this review, we are discussing and exploring the novel strategies [6, 7], specially focussing on Stem Cell based therapy (with and without immune cells), RNA, and DNA (Nucleic Acid) based vaccine trials so far used, or in process of implementations against COVID-19 pandemic.

\section{Key Novel Strategies During the Outbreak of COVID-19 Viral Disease}

This situation is like a new puzzle out in the open for all to solve. Since the first reported case of COVID-19 several anti-viral and antiparasite drugs were used but the efficacy of all such trials are questioned statistically. Hence we postulate and simultaneously hope for new modalities to have potential to manage this new viral pandemic and in our review this seems quite plausible in the near future.

\section{Stem Cell Therapy: Significance in Viral Disease (COVID-19)}

In recent years stem cell therapy has become one of the emerging therapeutic strategies in the treatment of many intractable diseases including viral infections. Although the development and progression in these cell-based therapies, especially pluripotent stem cells, are quite slow due to limited sources of its isolation and associated ethical constraints. The common cells of choice are mesenchymal stem cells (MSCs), may be due to source prospective or availability, a high multiplying rate, low invasive procedure, and relative less of ethical constraints [8,9].

Advantages of mesenchymal stem cell-based therapy include (1) easy accessibility and isolation from bone 
marrow (BM, most preferred site), peripheral blood (PB) and adipose tissues (abdominal fat, infrapatellar fat pad, and buccal fat pad). These cells can also be isolated easily from neonatal birth-associated tissues including placenta (PL), umbilical cord (UC), Warton jelly (WJ), amniotic fluid (AF), and cord blood (CB). These cells once isolated can then be stored for future possible applications; (2) the cells are multipotent stem cells, can self-renew by dividing and has ability to differentiate into multiple specialized cells; (3) MSCs can easily expand to required clinical and scientific volume in relatively shorter time period; (4) MSCs can be deposited for repetitive therapeutic applications; (5) clinical trials of MSCs until now have not revealed any severe adverse body reactions to allogeneic MSC; (6) safety of MSCs have been reported in many clinical trials [8].

Besides the availability, storage and safety, the efficacy of MSCs in viral diseases is also to be well explained theoretically. It is well established that during viral infections of high replicative and virulence, the innate and adaptive immunity defense get impaired. Orchestrated immune players especially APCs (Antigen Presenting Cell: Dendritic cells, Macrophages, etc.) fail to engulf, process, and present antigens to adaptive immune players ( $\mathrm{T}$ cells and $\mathrm{B}$ cells). The above failure in innate and ultimately in the adaptive immune system provides green signals to proliferate viruses and aggravate their infections, consequently the inflammation due to imbalance in cytokine milieu occurs like other infective diseases and cancer etc., [10-13].

Studies have shown that COVID-19 triggers mounting of aggravated cytokine storms, which is mainly projected due to a large number of inflammatory factors-interleukins and immune cells $[13,14]$. The cytokine storm generated ultimately impairs the APCs and adaptive immune cells. Thus nowadays, the researchers as shown in Table 1 are in a way to develop MSCs therapy with either natural APCs or with artificial APCs to mount an appropriate immune response.

As per previous studies on other viral diseases and few also on COVID-19, it has been shown that MSCs retains immune-regulatory potential by modifying immune responses with the help of proliferation and function of numerous immune cells, like, inhibiting differentiation of monocytes into dendritic cells (DCs), changing the cytokine profiles of DCs with upregulation of regulatory cytokines and suppression of inflammatory cytokines. MSCs are also known to induce tolerant phenotypes of naive and effector $\mathrm{T}$ cells, constraining antibody production by B cells, and suppressing NK cell proliferation and NK cell-mediated cytotoxicity [15-19]. These immunomodulatory activities are mediated by both cellcell communications and secreted cytokines, including
interferon-(IFN-) $\gamma$, indoleamine 2,3-dioxygenase (IDO), transforming growth factor-(TGF-) $\beta$, interleukin (IL-) 6 , IL-10, and prostaglandin E2 [19-23]. With these suggested advantages and in hope of successful management of this pandemic several trials are underway on MSCs by the United States, China, Israel, Iran, Italy, and Iraq, etc., to combat the outbreak of COVID-19 as shown in Table 1 .

In a case report of a 65-year-old female patient diagnosed with COVID-19, hospitalised in critical condition had a neutrophilia (increase of $87 \%$ ) and an associated lymphocytopenia (decrease of 9.8\%), was treated with antiviral drugs such as lopinavir/ritonavir, IFN- $\alpha$, and oseltamivir but the vital signs kept on worsening. As a trial of treatment patient was started on umbilical cord mesenchymal stem cells (UC-MSCs) with $\alpha 1$ thymosin $5 \times 10^{7}$ cells three times each, and close monitoring of vitals done. Serum parameters revealed that after the second injection, serum albumin, CRP, and ALT/AST gradually reduced, along with improvement in vital signs. Thereafter, the patient was weaned off gradually from life supporting devices. On investigations the number of total white blood cells and neutrophils returned back to a normal level, while the number of lymphocytes increased to their normal level. Further assessment revealed a significant increase in numbers of $\mathrm{CD} 3+\mathrm{T}$ cell, CD4+ $\mathrm{T}$ cell, and $\mathrm{CD} 8+\mathrm{T}$ cell. Radiological evidence of improvement was noted after a third dose of cord stem cells. Ultimately the patient was discharged from the ICU ward. This case report strongly recommended that umbilical cord mesenchymal stem cells alone or in combination with other immune modulators should be explored as a treatment option for acute COVID-19 patients [24].

Recently researchers from China (Beijing, Shanghai, and Hubei) completed a study in which they successfully transplanted human MSCs (Trial depicted in Table 1). The study showed improvement in the outcome of 7 recruited and treated patients with COVID-19 pneumonia in Beijing YouAn Hospital, China, from Jan 23, 2020, to Feb 16, 2020. The clinical results, as well as alterations of inflammatory expressions, immune function levels, and adverse effects of 7 registered patients were evaluated for 14 days after MSC injection. MSCs transplant significantly improved the symptoms and signs of all seven patients without any adverse effects. The pulmonary function tests of these patients were significantly better 2 days post MSC transplantation. After the transplant, the increase in peripheral lymphocytes counts was noticed and the C-reactive protein (infection and inflammatory marker) decreased. Additionally, exaggerated levels of cytokine secreting immune cells CXCR3+CD4+ $\mathrm{T}$ cells, CXCR3 + CD8 + T cells, and CXCR3 + NK cells seen initially vanished in 3-6 days post treatment. Further, a group of $\mathrm{CD} 14+, \mathrm{CD} 11 \mathrm{c}+, \mathrm{CD} 11$ and regulatory 


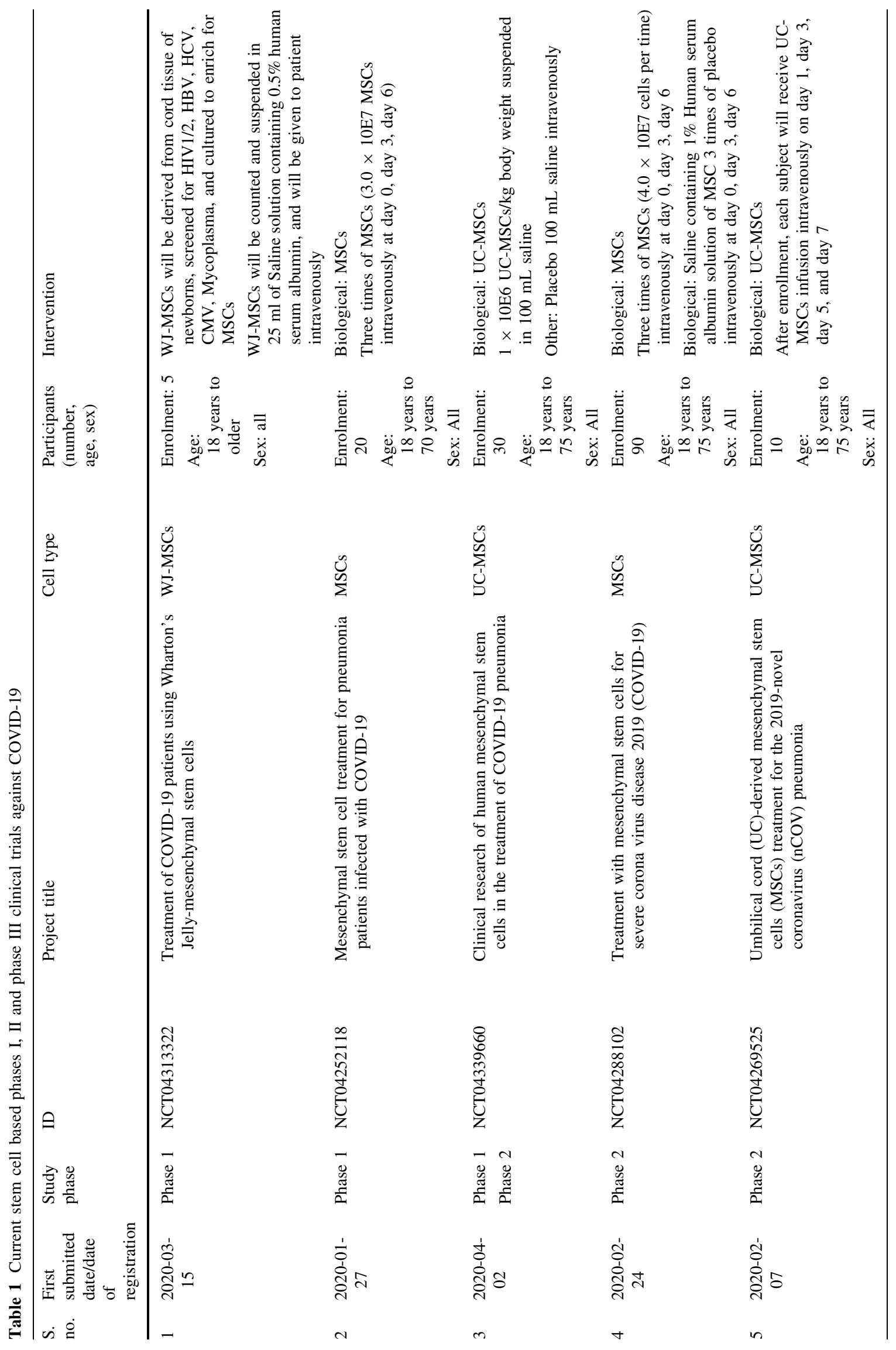




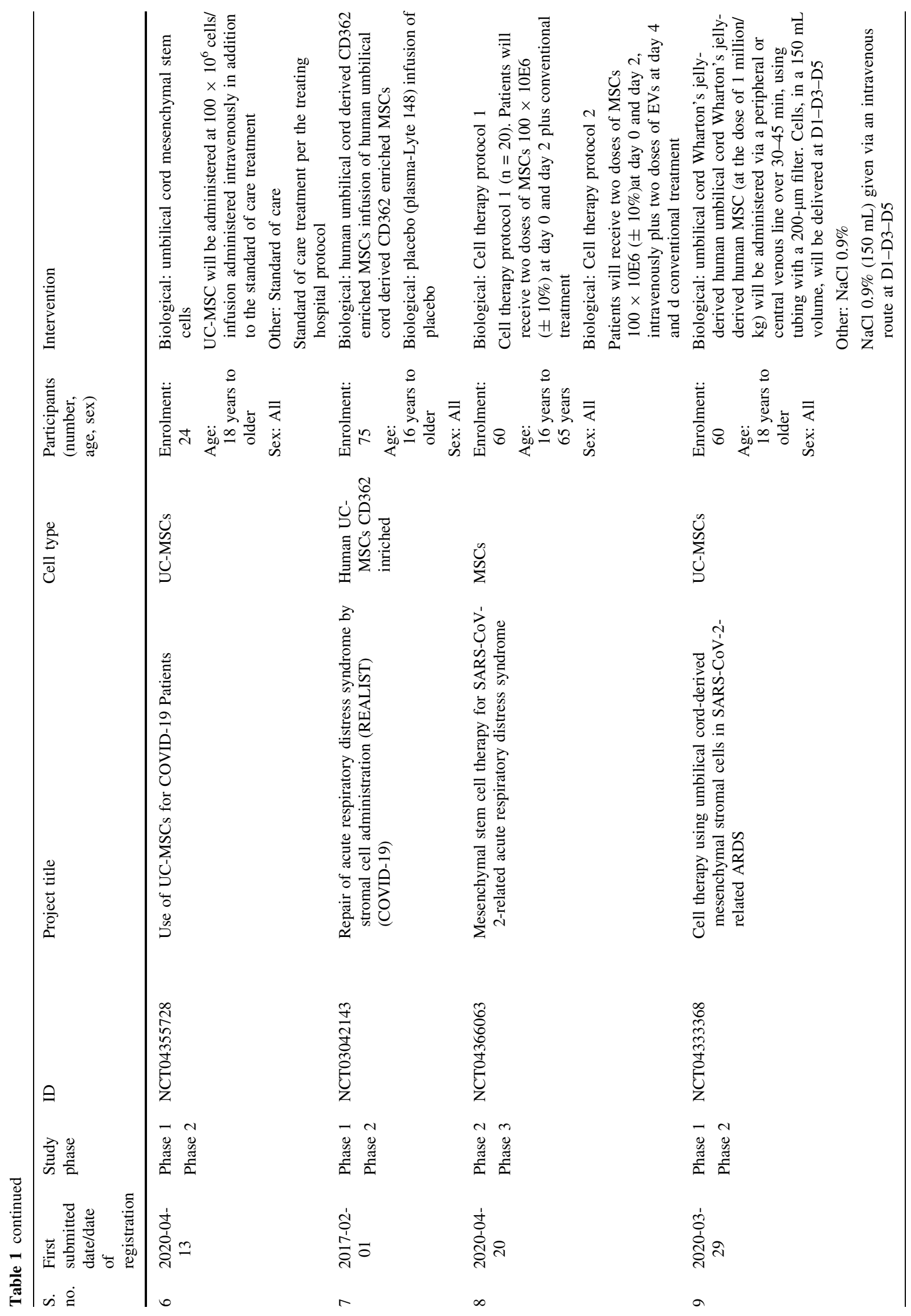




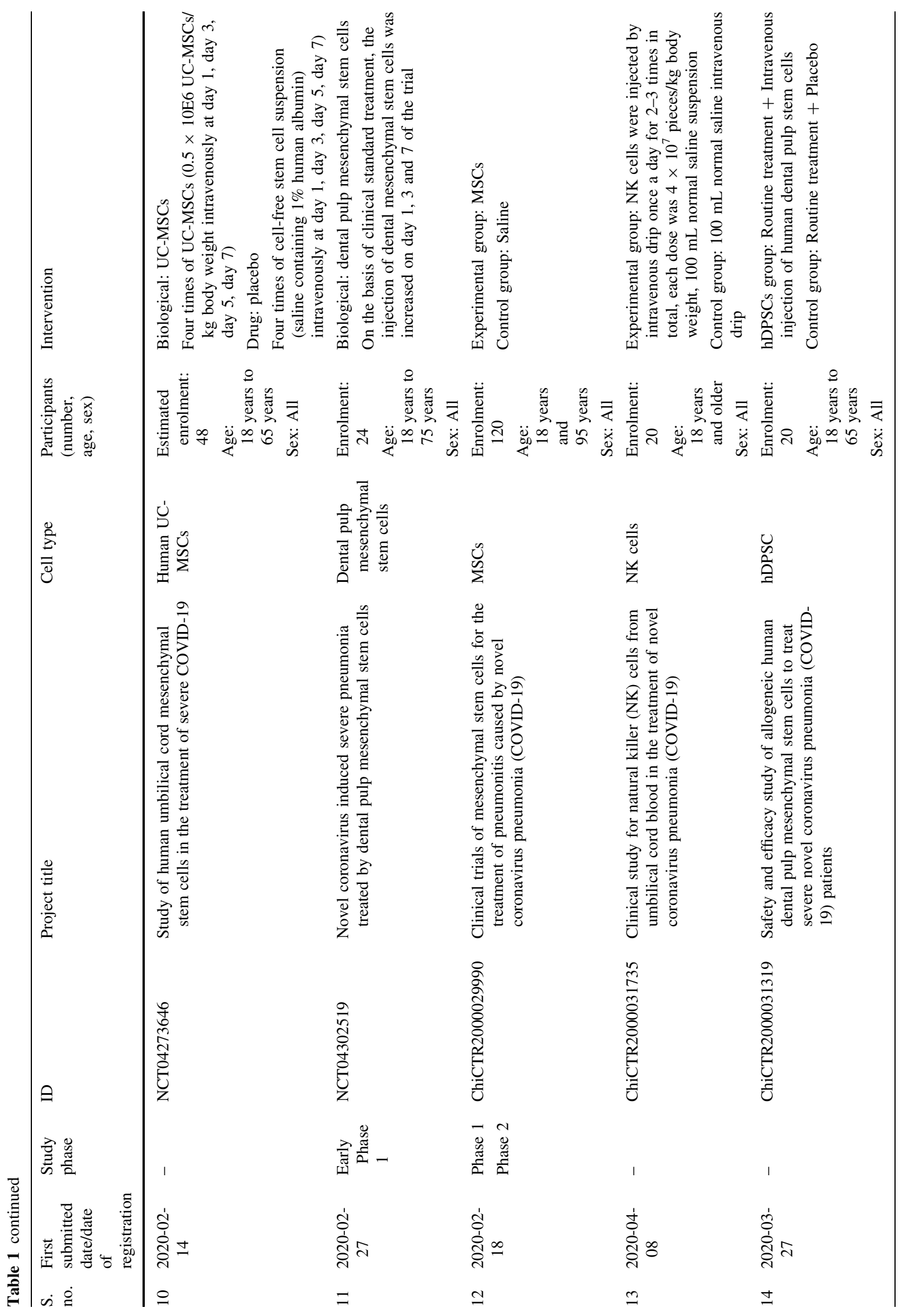




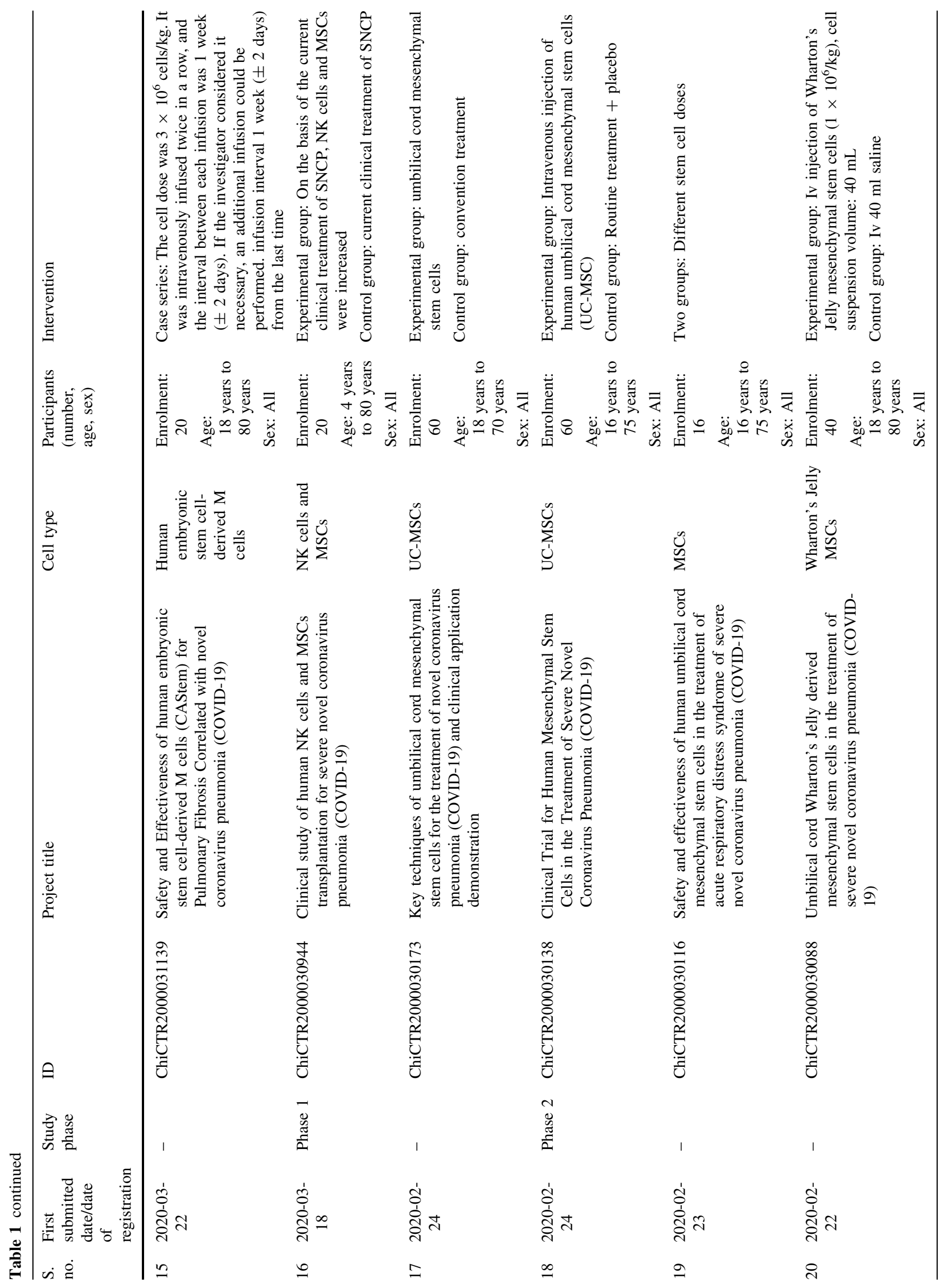




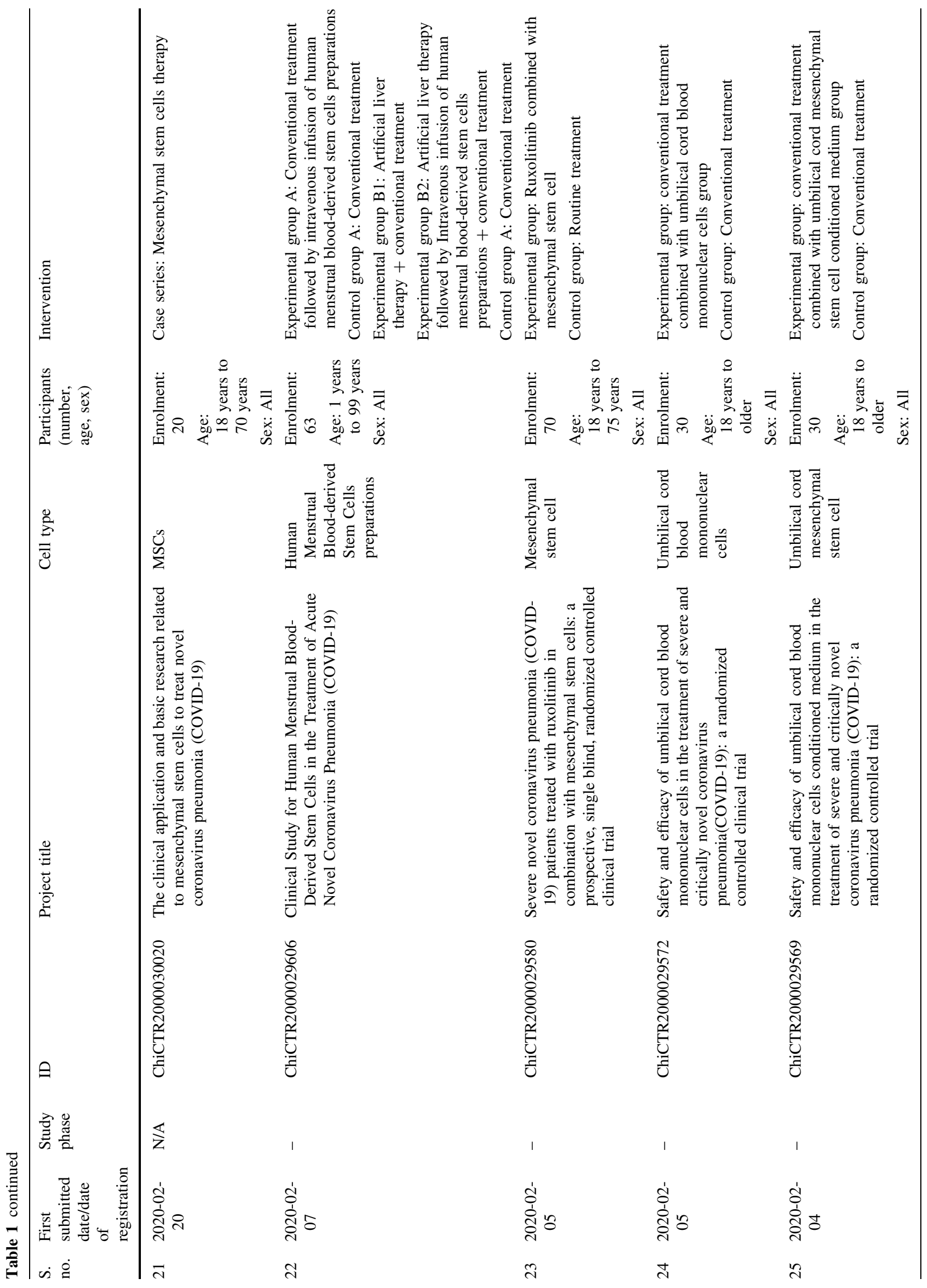


dendritic cells population was also increased. Along with these the level of pro-inflammatory TNF- $\alpha$ was seen to be decreased and anti-inflammatory IL-10 levels were increased significantly in MSC based treated group compared to the control placebo group. Thus, this very first study of intravenous transplantation of MSCs has opened a new vista as it was reported safe and effective for treatment in patients with COVID-19 pneumonia [25].

Further, one more trial of MSCs in March-April 2020 is reported by Pluristem Therapeutics Inc. (Nasdaq: PSTI) (TASE: PSTI) HAIFA, Israel. A leading regenerative medicine company has claimed successful treatment of patients suffering from COVID-19 complications in the United States. The treatment was as per the U.S. Food and Drug Administration's (FDA) Single Patient Expanded Access Program, also called a compassionate use program, which is part of the U.S. Coronavirus Treatment Acceleration Program (CTAP), an emergency program for that allows quick trials of all possible, promising and available modalities in effective management of the pandemic. Before treatment with PLX, the patient was reported as critically ill with respiratory failure due to acute respiratory distress syndrome (ARDS) and was on life support facilities for 3 weeks. As per company news bulletin, PLX cells are allogeneic mesenchymal-like cells that possess immunomodulatory properties and are able to induce the immune system's natural regulatory T cells and M2 macrophages and thus may check or reverse the hazardous aggravation of the immune system. So, PLX cells may potentially reduce the incidence andlor severity of COVID19 pneumonia and pneumonitis leading hopefully to a better prognosis for the patients. Now, the company is planning to work with the BIH Center for Regenerative Therapy (BCRT) and the Berlin Center for Advanced Therapies (BeCAT) at Charite' University of Medicine Berlin to explore PLX cell products candidates for Covid19.

As per the report on 8th May 2020, the company registered a Phase II trial after getting clearance from FDA titled "A Randomized, Double-Blind, Placebo-Controlled, Multicentre, Parallel-Group Phase II Study to Evaluate the Efficacy and Safety of Intramuscular Injections of PLXPAD for the Treatment of severe COVID-19". They proposed to treat 140 adult patients that are intubated and mechanically ventilated and are suffering from respiratory failure and ARDS due to COVID-19. The primary efficacy endpoint of the study is the number of ventilator-free days during the 28 days from day 1 through day 28 of the study [26].

The initial result of the above phase of trial was made available on May 14, 2020 in which the company claimed $87.5 \%$ survival rate of patients who were admitted with invasive mechanical ventilation. Out of these $75 \%$ of patients no longer require any mechanical ventilation. Further, $62.5 \%$ of the patients were discharged from the hospital.

In conclusion, the above trials of MSCs-based therapy in COVID-19 patients suggest that this is worth exploring option for treatment of novel coronavirus or any other similar outbreaks in the future.

\section{Synthetic Nano Stem Cells Based Therapy}

It has been reported that during viral infections including COVID-19, the dysregulated cytokine storm with enhanced expression of pro-inflammatory and downgraded expression of anti-inflammatory is a common phenomenon. In an animal-based study it is documented that leukaemia inhibitory factor (LIF) plays an essential role in neutralizing the cytokine storm in the lungs during viral pneumonia [27]. MSCs are known to release LIF, as per requirements but the cost burden of processing and maintaining it is on a higher side for the general patients. Answer to this comes with the invention of "LIFNano" which is a result of application of nanotechnology principles on generation of synthetic stem cells that has shown a thousand times more potency than soluble LIF [28, 29].

Although only a single finding of "LIFINano" i.e. Nanotechnology-based synthetic stem cells against experimental allergic encephalomyelitis (EAE) a preclinical model of multiple sclerosis has been claimed, where treatment with LIFNano reversed paralysis within 4 days, a timeline in accord with that reported for beneficial effects in COVID-19 pneumonia using MSC therapy. This study has shown that LIF is particularly important for the activation of epithelial STAT3 which is required for proper functioning of lung alveoli, and the treatment with exogenous LIF or LIF over-expression can limit pulmonary inflammation in response to LPS or hypoxia [29]. Thus, LIFNano has a cutting edge technology and it could be utilized as an alternative to cell-based therapy, to cater the need for a high volume and off-the-shelf therapeutic agents. Thus, this therapy may be able to rejuvenate injured tissues and suppress the cytokine storm in atypical pneumonia of COVID 19 patients.

\section{DNA/RNA Vaccines and COVID-19}

In the last 3 decades, an innovative approach of vaccination has been developed, which involves the direct introduction of a plasmid encompassing the DNA sequence encoding the antigen(s) into appropriate tissues in which an immune response is required. This method provides several prospective benefits over conventional approaches, such as 
the stimulation of both B- and T-cell responses, enhanced vaccine stability, the absence of any infectious entity, and the feasibility of large-scale production.

As evidence of the principle of DNA vaccination, immune reactions in animals have been assessed using genes from a diverse class of infectious agents, including influenza virus, hepatitis B virus, human immunodeficiency virus, rabies virus, lymphocytic choriomeningitis virus, malarial parasites, and mycoplasmas. In a few studies, protection from disease in animals has also been achieved. Though the worth and benefits of DNA vaccines must be evaluated on a case-by-case basis and their relevance will be determined by the nature of the agent being immunized against, the nature of the antigen, and the type of immune response required for protection.

The arena of DNA vaccination is evolving rapidly. Vaccines presently being developed utilizes not only DNA, but also encompasses an accessory segment that supports DNA to enter precisely into targeted cells, or that may work as adjuvants in exciting or directing the immune response. The earliest DNA vaccines approved for marketing are likely to use plasmid DNA derived from bacterial cells. There are many gene-based vaccines permitted for use in veterinary practice for targeting canine melanoma (Merial), West Nile virus (Wyeth), fish hematopoietic necrosis virus (Novartis), and swine growth hormonereleasing hormone (Inovio). Now, diverse strategies are in process to execute with RNAs as well as with complexes of nucleic acid molecules and other entities [30].

It is recognized that the development and application of nucleic acid vaccines are evolving rapidly. Currently, during the COVID-19 outbreak, several DNA and RNA vaccines trials as depicted in Tables 2 and 3 are also in progress, few are in clinical phase I and II trials while others are in the pre-clinical validation stage by various institutions and pharmaceutical companies. Like initial initiative in Feb 2020 as DNA vaccine was taken by Applied DNA Sciences USA, subsidiary LineaRx and Takis Biotech Italy, by establishing a joint venture to develop a linear DNA vaccine as a treatment for coronavirus. The vaccine development utilizes Polymerase Chain Reaction (PCR)-based DNA manufacturing technology to develop the vaccine.

Another clinical trial of DNA Vaccines has been initiated by Inovio Pharmaceuticals, USA in collaboration with Coalition for Epidemic Preparedness Innovations (CEPI), Norway. The current open-label phase I trial has been initiated in 40 participants to evaluate the safety, tolerability, and immunological profile of INO-4800 administered by intradermal (ID) injection followed by electroporation (EP) using the CELLECTRA ${ }^{\circledR} 2000$ device in healthy adult volunteers.
Similarly, a RNA based vaccine's phase I trial is in the process by the National Institute of Allergy and Infectious Diseases (NIAID) United States, registered trial as Safety and Immunogenicity Study of 2019-nCoV Vaccine (mRNA-1273) for Prophylaxis of SARS-CoV-2 Infection (COVID-19) on 105 participants. Recently, Entos Pharmaceuticals USA, is developing Fusogenix DNA vaccine by using the Fusogenix drug delivery platform to prevent COVID-19 infections. Fusogenix drug delivery platform is a proteo-lipid vehicle that introduces genetic payload directly into the cells. Entos is working on developing an optimized payload containing multiple protein epitopes derived from SARS-COV-2 proteins, which will stimulate an immune response in the body to prevent COVID-19 infection. Similarly, Zydus Cadila India, declared the preclinical trial to develop a vaccine for COVID-19 using two novel approaches. The first approach includes the development of a DNA vaccine against the viral membrane protein of the virus, while a live attenuated recombinant measles virus (rMV) vectored vaccine will be developed in the other. Further, several preclinical trials on DNA/RNA based vaccines have been started globally by reputed institute/University like Karolinska Institute Sweden, Waterloo University Canada, Fudan University China, Shanghai JiaoTong University China to pharmaceutical company like Zydus cadila India, BioNet Asia Immunomic Therapeutics Thai-French privately-held biotech company etc., and many more as shown in Tables 2 and 3. These institutions/Universities and Pharmaceutical industries are in the process of developing suitable DNA/RNA based vaccines against the COVID-19 virus at the earliest [31].

\section{Conclusion}

The results of these on-going clinical trials on new therapeutic options have brought in hope in controlling the spread of novel coronavirus and sufferings of patients with COVID-19. Not only this, the success of such trials may prove the best utilization of the technological advances and their integration with health sciences. RNA and DNA vaccines offer us the potential for more rapid vaccine development because synthetic RNA and DNA are easier to construct and are relatively purified from contaminants (improving safety) with immense potential of scaling up the volume of production in comparison to the traditional vaccines. As mentioned in text, the Phase I and Phase II clinical trials of Pluristem Therapeutics Inc. (Nasdaq: PSTI) (TASE: PSTI) HAIFA, Israel, working on mesenchymal stem cells for treating COVID-19 patients has shown higher recovery rate and less mortality paving the way to explore this treatment strategy for the large number of patients affected with severe forms of COVID-19 all 


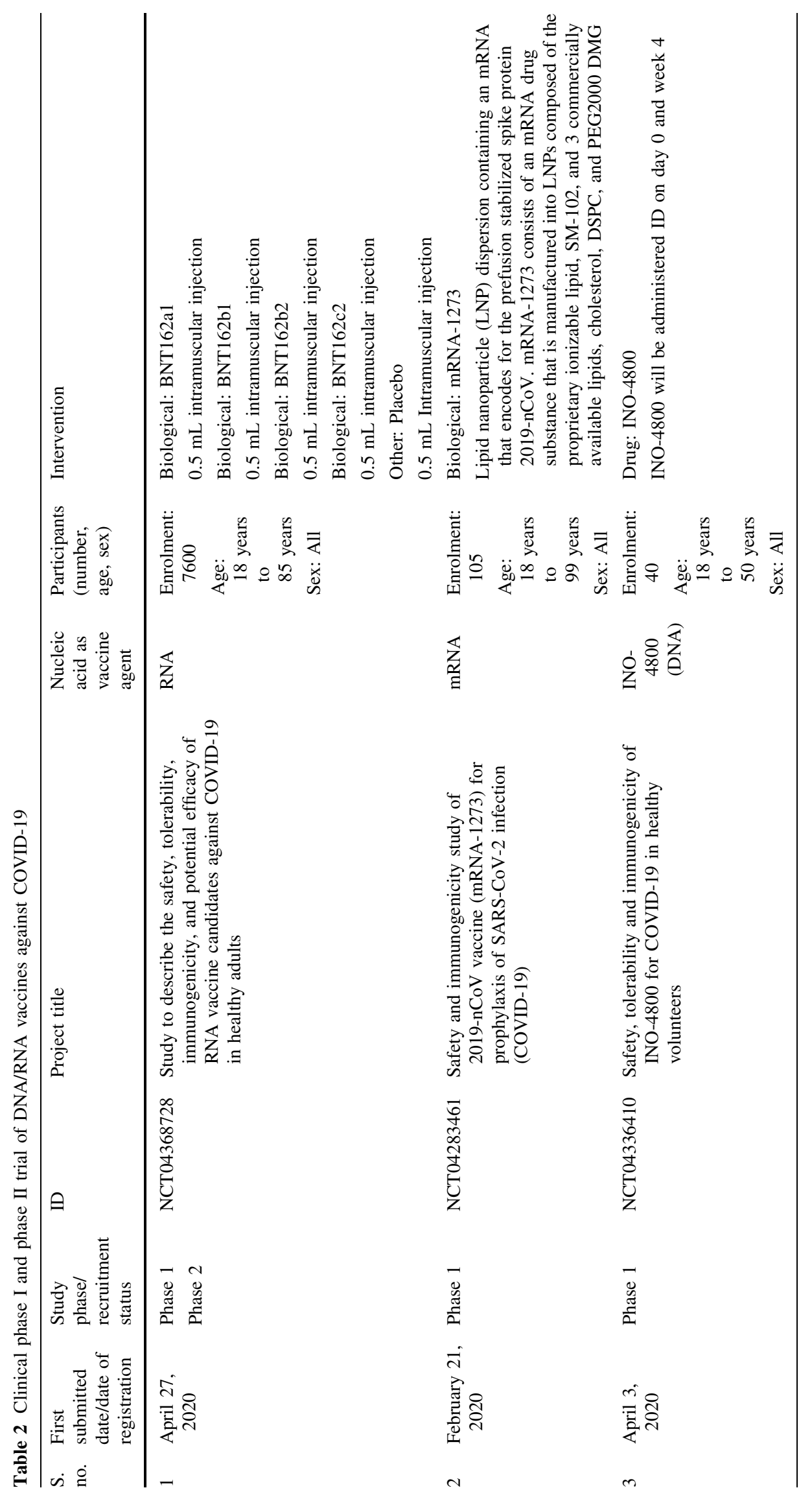


Table 3 Pre-clinical trial ${ }^{\mathrm{a}}$ on DNA/RNA vaccines against Covid-19

\begin{tabular}{|c|c|c|c|c|c|}
\hline $\begin{array}{l}\text { S. } \\
\text { no. }\end{array}$ & Platform & Candidate vaccine & Developer & $\begin{array}{l}\text { Target } \\
\text { diseases }\end{array}$ & $\begin{array}{l}\text { Current } \\
\text { stage of } \\
\text { clinical } \\
\text { evaluation }\end{array}$ \\
\hline 1. & DNA & DNA & $\begin{array}{l}\text { Takis Italy/Applied DNA Sciences USA/Evvivax, Czech } \\
\text { Republic }\end{array}$ & COVID-19 & Pre-clinical \\
\hline 2. & DNA & Plasmid DNA, needle-free delivery & $\begin{array}{l}\text { Immunomic Therapeutics Inc./EpiVax, Inc./PharmaJet, } \\
\text { Inc.USA }\end{array}$ & COVID-19 & Pre-clinical \\
\hline 3. & DNA & DNA plasmid vaccine & Zydus Cadila, India & COVID-19 & Pre-clinical \\
\hline 4. & DNA & DNA vaccine & BioNet Asia, Thai-French biotech company & COVID-19 & Pre-clinical \\
\hline 5. & DNA & DNA vaccine & University of Waterloo & COVID-19 & Pre-clinical \\
\hline 6. & RNA & $\begin{array}{l}\text { LNP-encapsulated mRNA cocktail } \\
\text { encoding VLP }\end{array}$ & $\begin{array}{l}\text { Fudan University/Shanghai JiaoTong University/ } \\
\text { RNACure Biopharma, China }\end{array}$ & COVID-19 & Pre-clinical \\
\hline 7. & RNA & $\begin{array}{l}\text { LNP-encapsulated mRNA encoding } \\
\text { RBD }\end{array}$ & $\begin{array}{l}\text { Fudan University/Shanghai JiaoTong University/ } \\
\text { RNACure Biopharma, China }\end{array}$ & COVID-19 & Pre-clinical \\
\hline 8. & RNA & $\begin{array}{l}\text { Replicating defective SARS-CoV-2 } \\
\text { derived RNAs }\end{array}$ & Centro Nacional Biotecnología (CNB-CSIC), Spain & COVID-19 & Pre-clinical \\
\hline 9. & RNA & LNP-encapsulated mRNA & University of Tokyo/Daiichi-Sankyo, Japan & COVID-19 & Pre-clinical \\
\hline 10. & RNA & Liposome-encapsulated mRNA & BIOCAD, Russia & COVID-19 & Pre-clinical \\
\hline 11. & RNA & mRNA & China CDC/Tongji University/Stermina, China & COVID-19 & Pre-clinical \\
\hline 12. & RNA & mRNA & Arcturus/Duke-NUS, Singapore & COVID-19 & Pre-clinical \\
\hline 13. & RNA & mRNA & BioNTech Germany/Fosun Pharma China/Pfizer USA & COVID-19 & Pre-clinical \\
\hline 14. & RNA & siRNA & Imperial College London, UK & COVID-19 & Pre-clinical \\
\hline 15. & RNA & mRNA & Curevac, Germany & COVID-19 & Pre-clinical \\
\hline
\end{tabular}

${ }^{a}$ Data obtained from institutions/University's website and Pharmaceutical Company's website and https://www.who.int/blueprint/priority-dis eases/key-action/novel-coronavirus-landscape-ncov.pdf

over the world. MSCs are well known in downgrading the inflammation and activation of Antigen Presenting Cells, but there is no evidence of their role in the activation of $\mathrm{B}$ cells and T cells; which is well-known role of DNA and RNA based vaccines, so both MSCs and Nucleic acid based vaccines in a synergistic way or with immuneengineered modification, may produce better results in combating with this virus $[32,33]$. Thus utilization of such novel vaccines might be a better way for mitigation of the current COVID-19 pandemic. The finding that mesenchymal stem cells and DNA/RNA vaccines are safe and can reverse severe critical disease with high potency is a key breakthrough representing an entirely new biological approach to treatment of such deadly viral diseases. Given the pressing needs to keep mortality as low as possible, such treatments need to be researched and developed promptly.

\section{Future Prospective}

Remarkably, after 3 decades of discovery of DNA vaccines, and after few unsatisfactory human clinical trials of first-generation vaccines, DNA vaccines are now undergoing advancement with the introduction of more competent designs, isolations of bio-molecules, characterizations and better delivery technologies including electroporation. Like, the past of monoclonal antibody vaccines development programme, initially went through a highly negative phase but now has emerged as pharmaceutical blockbusters, this also has scalable potential over the others. Now it is believed that DNA vaccines have passed their darkest phase and thereby will soon be ready to emerge as commercially viable products, most likely in the area of antiviral vaccines. Similarly, the MSC platform has the potential to express hundreds of peptides, thereby generating a broad array of epitopes with precise posttranslational processing, mimicking natural infection. Thus, by inducing immunity to a combination of epitopes and complimentary determining regions (CDRs), it may be possible to develop prophylactic and even therapeutic vaccines against current pandemic disease COVID-19, where traditional vaccination approaches have failed to solve the puzzle. We hope that these innovative vaccine strategies may open new vistas in the management of COVID-19 and soon whole humanity would be free from the current COVID-19 viral outbreak. 


\section{Compliance with Ethical Standards}

Conflict of interest The authors declare that they have no conflict of interest.

Ethical Approval As present article is a systematic review of the study already done or in process by other researchers, so there is no need to have such clarifications.

\section{References}

1. Mitra P, Misra S, Sharma P. COVID-19 pandemic in India: what lies ahead. Ind J Clin Biochem. 2020. https://doi.org/10.1007/ s12291-020-00886-6.

2. Ren LL, Wang YM, Wu ZQ, Xiang ZC, Guo L, Xu T, et al. Identification of a novel coronavirus causing severe pneumonia in human: a descriptive study. Chin Med J. 2020;133(9):1015-24.

3. Ministry of Health and Family Welfare COVID-19 INDIA (2020) https://www.mohfw.gov.in/.

4. Coronavirus Disease (2019) World Health Organization. https:// www.who.int/health-topics/coronavirus\#tab=tab_3. Accessed 25 May 2020.

5. Dwivedi S, Yadav SS, Singh MK, Shukla S, Khattri S, Pant KK. Pharmacogenomics of viral diseases. In: Barh D, Dhawan D, Ganguly N, editors. Omics for personalized medicine. New Delhi: Springer; 2013. p. 637-76.

6. Pan L, Mu M, Yang P, Sun Y, Wang R, Yan J, et al. Clinical characteristics of COVID-19 patients with digestive symptoms in Hubei, China: a descriptive, cross-sectional, multicenter study. Am J Gastroenterol. 2020;115(5):766-73.

7. Sharma P, Dwivedi S. Prospects of molecular biotechnology in diagnostics: step towards precision medicine. Indian J Clin Biochem. 2017;32(2):121-3.

8. Golchin A, Farahany TZ, Khojasteh A, Soleimanifar F, Ardeshirylajimi A. The clinical trials of mesenchymal stem cell therapy in skin diseases: an update and concise review. Curr Stem Cell Res Therapy. 2018;14(1):22-33.

9. Dwivedi S, Sharma P. Stem cell biology: a new hope in regenerations and replenishments therapy. Indian $\mathrm{J}$ Clin Biochem. 2018;33(4):369-71.

10. Cronkite DA, Strutt TM. The regulation of inflammation by innate and adaptive lymphocytes. J Immunol Res. 2018;2018:1467538. https://doi.org/10.1155/2018/1467538.

11. Dwivedi S, Singh S, Goel A, Khattri S, Mandhani A, Sharma P, Misra S, Pant KK. Pro-(IL-18) and anti-(IL-10) inflammatory promoter genetic variants (intrinsic factors) with tobacco exposure (extrinsic factors) may influence susceptibility and severity of prostate carcinoma: a prospective study. Asian Pac J Cancer Prev. 2015;16(8):3173-81.

12. Dwivedi S, Shukla KK, Gupta G, Sharma P. Non-invasive biomarker in prostate carcinoma: a novel approach. Indian J Clin Biochem. 2013;28(2):107-9.

13. Mehta P, Mcauley DF, Brown M, Sanchez E, Tattersall RS, Manson J. COVID-19: consider cytokine storm syndromes and immunosuppression. Lancet. 2020;6736(20):19-20.

14. Thanunchai M, Hongeng S, Thitithanyanont A. Mesenchymal stromal cells and viral infection. Stem Cells Int. 2015;2015:860950. https://doi.org/10.1155/2015/860950.

15. Aggarwal S, Pittenger MF. Human mesenchymal stem cells modulate allogeneic immune cell responses. Blood. 2005;105(4):1815-22.

16. Corcione A, Benvenuto F, Ferretti E, Giunti D, Cappiello V, Cazzanti $F$, et al. Human mesenchymal stem cells modulate B-cell functions. Blood. 2006;107(1):367-72.
17. Jiang XX, Zhang Y, Liu B, Zhang SX, Wu Y, Yu XD, et al. Human mesenchymal stem cells inhibit differentiation and function of monocyte-derived dendritic cells. Blood. 2005;105(10):4120-6.

18. Rasmusson I, Uhlin M, Le Blanc K, Levitsky V. Mesenchymal stem cells fail to trigger effector functions of cytotoxic $\mathrm{T}$ lymphocytes. J Leukoc Biol. 2007;82(4):887-93.

19. Spaggiari GM, Capobianco A, Becchetti S, Mingari MC, Moretta L. Mesenchymal stem cell-natural killer cell interactions: evidence that activated NK cells are capable of killing MSCs, whereas MSCs can inhibit IL-2-induced NK-cell proliferation. Blood. 2006;107(4):1484-90.

20. Krampera M, Cosmi L, Angeli R, Pasini A, Liotta F, Andreini A, et al. Role for interferon-gamma in the immunomodulatory activity of human bone marrow mesenchymal stem cells. Stem Cells. 2006;24(2):386-98.

21. Meisel R, Zibert A, Laryea M, Göbel U, Däubener W, Dilloo D. Human bone marrow stromal cells inhibit allogeneic T-cell responses by indoleamine 2,3-dioxygenase-mediated tryptophan degradation. Blood. 2004;103(12):4619-21.

22. Németh K, Leelahavanichkul A, Yuen PS, Mayer B, Parmelee A, Doi $\mathrm{K}$, et al. Bone marrow stromal cells attenuate sepsis via prostaglandin $\mathrm{E}(2)$-dependent reprogramming of host macrophages to increase their interleukin-10 production. Nat Med. 2009;15(1):42-9. https://doi.org/10.1038/nm.1905.

23. Tse WT, Pendleton JD, Beyer WM, Egalka MC, Guinan EC. Suppression of allogeneic T-cell proliferation by human marrow stromal cells: implications in transplantation. Transplantation. 2003;75(3):389-97.

24. Bing Liang, Junhui Chen, Tao Li, Haiying Wu, Wenjie Yang, Yanjiao Li, J, et al. Clinical remission of a critically ill COVID19 patient treated by human umbilical cord. ChinaXiv 2020. https://doi.org/10.12074/202002.00084.

25. Leng Z, Zhu R, Hou W, Feng Y, Yang Y, Han Q, et al. Transplantation of ACE2-mesenchymal stem cells improves the outcome of patients with COVID-19 pneumonia. Aging Dis. 2020;11(2):216-28.

26. U.S. FDA Clears Pluristem's IND Application for Phase II COVID-19 Study. https://www.pluristem.com/wp-content/ uploads/2020/05/FDA-Clearance-COVID-19-FINAL.pdf. Accessed 25 May 2020.

27. Foronjy RF, Dabo AJ, Cummins N, Geraghty P. Leukemia inhibitory factor protects the lung during respiratory syncytial viral infection. Immunology. 2014;15:41.

28. Metcalfe SM, Strom TB, Williams A, Fahmy TM. Multiple sclerosis and the LIF/IL- 6 axis: use of nanotechnology to harness the tolerogenic and reparative properties of LIF. Nanobiomedicine. 2015;2:5.

29. Metcalfe SM. Mesenchymal stem cells and management of COVID-19 pneumonia. Med Drug Discov. 2020;5:100019. https://doi.org/10.1016/j.medidd.2020.100019.

30. Dwivedi S, Purohit P, Sharma P. MicroRNAs and diseases: promising biomarkers for diagnosis and therapeutics. Ind J Clin Biochem. 2019;34(3):243-5.

31. WHO. https://www.who.int/blueprint/priority-diseases/key-action/ novel-coronavirus-landscape-ncov.pdf. Accessed 25 May 2020.

32. Dwivedi S, Purohit P, Misra R, Pareek P, Goel A, Khattri S, et al. Diseases and molecular diagnostics: a step closer to precision medicine. Indian J Clin Biochem. 2017;32(4):374-98.

33. Dwivedi S, Purohit P, Mittal Y, Gupta G, Goel A, Verma RC, et al. Genetic engineering: towards gene therapy and molecular medicine. In: Arh D, Azevedo V, editors. Omics technologies and bio-engineering: towards improving quality of life. Elsevier: Academic Press; 2017. p. 507-30.

Publisher's Note Springer Nature remains neutral with regard to jurisdictional claims in published maps and institutional affiliations. 